\title{
An Overview and Efficiency Analysis of Dominant Frequencies Estimation Methods with the Application of DIRECT Global Minimization Method
}

\author{
Katarina PISAČIĆ* Z Zlatko BOTAK, Marko HORVAT
}

\begin{abstract}
This report discusses the application of the following methods that are used to calculate maximum frequency of a spectrum: parabolic, modified parabolic by Rife and Vincent, the method according to Quinn from 1994 and the method according to Quinn from 1997. After introducing DIRECT global minimization method, the results obtained using previously mentioned methods are compared with the results obtained using DIRECT global minimization of the Lipschitz function. Determining vibration frequency and comparing to previously collected data or knowledge base data is a common procedure. In engineering practice it is often necessary to determine not one, but several so-called dominant frequencies or local maxima; the procedure is sometimes called multi peak detection and here we propose an algorithm for computerised multi peak detection.
\end{abstract}

Keywords: DFT, Discrete Fourier transformation, DIRECT global minimization, dominant frequency, Lipschitz, multi peak detection, spectral analysis, vibration

\section{INTRODUCTION}

Monitoring vibration levels is an effective method of forecasting and detecting mechanical failures in rotating components of mechanical systems. Measurements are carried out periodically using hand held portable devices or continuously by means of stationary systems. Measured vibration level may indicate irregularities caused by incorrect assembly even before the occurrence of failure [1]. Ageing of system mechanical components, appearance of wear and minor damage will increase the level of amplitude of certain frequencies; therefore first step in fault detection is interpretation of the measured amplitude and frequency of vibration. Measured data can be used to determine the condition of a mechanical component in exploitation, predict the occurrence of damage and identify type of fault based on the analysis of measured vibration. In cases where there are no specially adapted stationary systems that monitor vibrations of equipment, maintenance staff often uses a visual method to compare measured data and knowledge base that contains spectrum forms of vibrations caused by various damages, classified by type of rotating components and the type of damage.

During visual comparison it is necessary to note and determine any dominant frequencies and their amplitudes. The measured data in time domain is converted to the frequency domain, using Discrete Fourier transform i.e. frequency spectrum is obtained from amplitudes measured in a given period of time using the discrete Fourier transform.

Values of the dominant frequency, phase and amplitude are obtained from the spectrum using one of the known algorithms; see for example [2, 3].

\section{INITIAL ASSUMPTIONS}

Assume measuring vibration of the electro motor housing, which occurs as a result of its operation. Let $\left(t_{n}\right.$, $\left.y_{n}\right), n=0, \ldots, m$ be measured data, while $t_{n}$ is time range, (usually equidistant range of values) on $y_{n}$ corresponding measured displacement values of a fixed point on an operating motor housing, directed as one unit vector.
Let $L>0, m \in \mathbb{N}$ and $\Delta t=\frac{L}{m}$. Assume measured data $\left(t_{n}, y_{n}\right), n=0, \ldots, m-1$, can be written

$t_{n}=n \Delta t$

$y_{n}=a \cos (2 \pi f n \Delta t+\varphi)+\varepsilon_{n}$

$n=0, \ldots, m-1$

while $a, f>0, \varphi \in[0,2 \pi\rangle$ are unknown parameters, and $\varepsilon_{n} \sim N\left(0, \sigma^{2}\right)$ (normally distributed random error with expectation 0 and variance $\sigma^{2}$ ). Additionally assume $m>>2 f \cdot L$. Parameter $f$ is named dominant frequency, parametar $a$ amplitude and $\varphi$ phase.

Let $A_{w}:\left[0, \frac{1}{2 \Delta t}\right] \rightarrow \mathbb{R}$ be function defined as follows

$A_{w}=\frac{1}{m}\left|\sum_{n=0}^{m-1} w_{n} y_{n} \varepsilon^{-2 n \pi i f \Delta t}\right|^{2}$

$A_{w}=\frac{1}{m}\left(\left(\sum_{n=0}^{m-1} w_{n} y_{n} \cos (2 \pi f n \Delta t)\right)^{2}+\left(\sum_{n=0}^{m-1} w_{n} y_{n} \sin (2 \pi f n \Delta t)\right)^{2}\right)$

where $w_{n}=w(n \Delta t)$, and $w:[0, L] \rightarrow[0,+\infty\rangle$ is window function for which applies $w(t)>0, t \in[0, L]$ and $w(t)=0, t \notin[0, L]$. Function $A_{w}$ is known in the literature as the periodogram [4] with window function $w$, assigned to data $\left(t_{n}, y_{n}\right), n=0, \ldots, m-1$.

We observe the following optimization problem

$f_{w}^{*}=\arg \max A_{w}(f)$

Assuming $m$ is large enough, global maximum $f_{w}^{*}$ of function $A_{w}$ on interval $\left[0, \frac{1}{2 \Delta t}\right]$ is used as estimator for 
dominant frequency. While amplitude ${a_{w}}^{*}$ and phase $\varphi_{w}{ }^{*}$ can be determined from the following expressions [5]:

$$
\begin{aligned}
& a_{w}{ }^{*} \cos \varphi_{w}{ }^{*}=\frac{2}{m} \sum_{n=0}^{m-1} w_{n} y_{n} \cos 2 \pi f_{w}^{*} n \Delta t, \\
& a_{w}{ }^{*} \sin \varphi_{w}{ }^{*}=\frac{2}{m} \sum_{n=0}^{m-1} w_{n} y_{n} \sin 2 \pi f_{w}{ }^{*} n \Delta t
\end{aligned}
$$

Usually window function is a rectangular window [6] defined as

$w_{R}(t)=\left\{\begin{array}{l}1, t \in[0, L] \\ 0, \text { otherwise }\end{array}\right.$ in vibration analysis is often used Hanning window [6], defined as

$$
w_{H}(t)=\left\{\begin{array}{l}
\frac{1}{2}\left(1-\cos \frac{2 \pi}{(m-1) \Delta t} t\right), t \in[0, L] \\
0, \text { otherwise }
\end{array}\right.
$$

Figure shows given data at $\left(t_{n}, y_{n}\right), n=0, \ldots, m$ generated based on parameter $a=11, f=4, \varphi=\frac{\pi}{2}, m=40$, $L=\pi$ and $\sigma=0.5$, effect of window function on given data and corresponding periodogram.

\section{Data Window function}
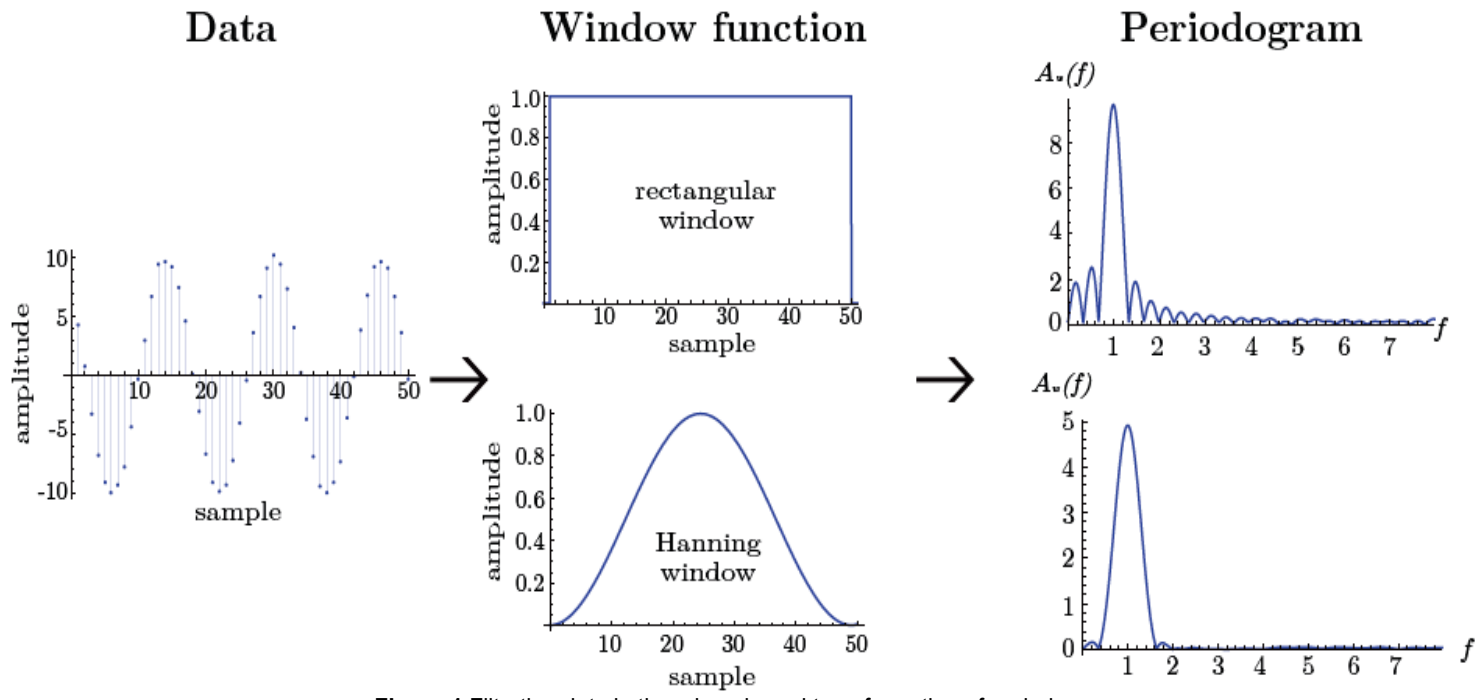

Figure 1 Filtrating data in time domain and transformation of periodogram

From numerical aspect the problem (6) is extremely complex as the function $A_{w}$ has a large number of local extrema, and this number is growing by increasing data quantity $m$. In the literature, various procedures can be found on local approximation of periodogram $A_{w}$ aiming to find a global maximum of the function $A_{w}$. In this article overview of some such functions is given and using numerical experiment their efficiency is examined, Furthermore it is shown that function $A_{w}$ is a Lipschitz class function and for the solution of problem (6) can be used DIRECT method for global optimization of Lipschitz functions [7-9]. Such obtained estimator is compared to other methods estimators and its efficiency is determined. Also in order to hasten estimation process DIRECT method is combined with a local method to narrow maximization scope and it will be determined if results can be enhanced comparing the DIRECT method with the mentioned local approximation methods. Finally, based on the numerical experiment findings the method for determining dominant frequencies will be proposed.

\section{OVERVIEW OF LOCAL APPROXIMATION METHODS FOR THE DETERMINATION OF DOMINANT FREQUENCIES}

Within this section an overview will be given of several methods used to determine dominant frequencies based on periodogram approximation.
Assuming given measurement data is $\left(t_{n}, y_{n}\right), n=0, \ldots$, $m-1$, window function, corresponding periodogram $A_{w}$, let us define function $I_{w}:\left[0, \frac{1}{2 \Delta t}\right] \rightarrow R$ such that

$$
I_{w}(f)=\sqrt{\frac{1}{m} A_{w}(f)}
$$

Notice

$$
f_{w}^{*}=\underset{f \in\left[0, \frac{1}{2 \Delta t}\right]}{\arg \max } I_{w}(f)=\underset{f \in\left[0, \frac{1}{2 \Delta t}\right]}{\arg \max } A_{w}(f)
$$

Function $I_{w}$ will be called modified periodogram.

\subsection{Discretization of Modified Periodogram}

We consider values of modified periodogram $I_{w}\left(f_{k}\right)$ on the discrete set of values $f_{k}=\frac{k}{m \Delta t}, k=0, \ldots,\left[\frac{m-1}{2}\right]$. Notice:

$$
I_{w}\left(f_{k}\right)=\frac{1}{m}\left|\sum_{n=0}^{m-1} w_{n} y_{n} \varepsilon^{-2 n \pi i \frac{k}{m \Delta t} \Delta t}\right|,
$$




$$
\begin{aligned}
& I_{w}\left(f_{k}\right)=\frac{1}{m} \mid \sum_{n=0}^{m-1} w_{n} y_{n} \varepsilon^{\frac{-2 n k \pi i}{m} \mid,} \\
& I_{w}\left(f_{k}\right)=\left|F\left[w_{0} y_{0}, \ldots, w_{m-1} y_{m-1}\right]\right|,
\end{aligned}
$$

while $F\left[w_{0} y_{0}, \ldots, w_{m-1} y_{m-1}\right]$ is discrete Fourier transformation of range $\left\{w_{0} y_{0}, \ldots, w_{m-1} y_{m-1}\right\}[10]$.

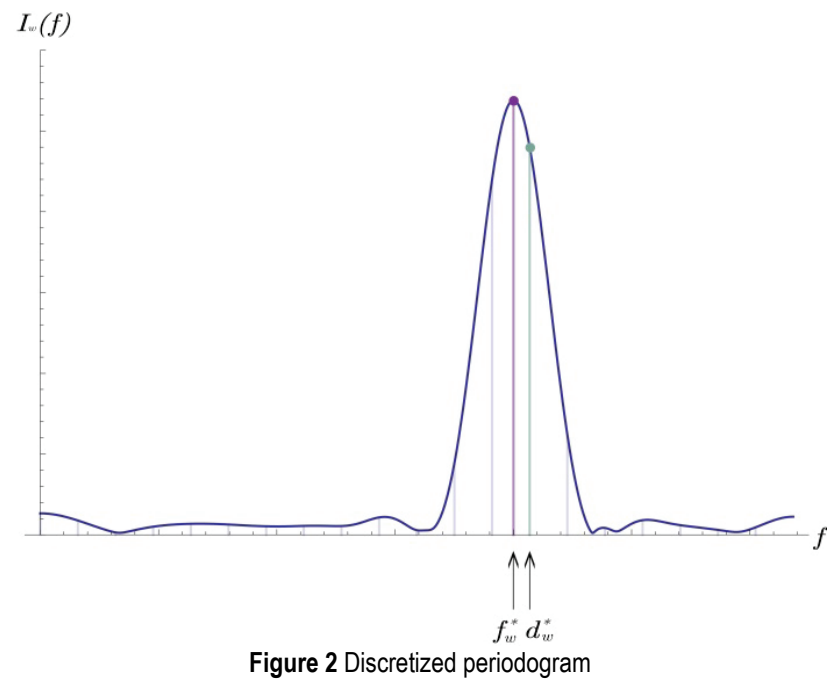

Let $k^{*} \in\left\{0, \ldots,\left[\frac{m-1}{2}\right]\right\}$ index such that

$$
\begin{aligned}
& I_{w}\left(f_{k^{*}}\right)=\max _{n=0, \ldots,\left[\frac{m-1}{2}\right]} I_{w}\left(f_{k}\right) \\
& f_{K}=\frac{k}{m \Delta t}, k=0, \ldots,\left[\frac{m-1}{2}\right]
\end{aligned}
$$

According to this approach maximization problem of real function of real variable is reduced to maximization problem of discrete set of values and therefore for approximation of global maximum $f_{w}^{*}$ of function, is adopted

$$
d_{w}^{*}=f_{k^{*}}=k^{*} \frac{1}{m \Delta t}
$$

Approximation of amplitude and matching phase is obtained from system of Eq. (7) and (8). While it is fair to expect that such an approximation will not be good enough (Fig. 2), point $d_{w} *$ is useful while global maximum $f_{w} *$ of function $I_{w}$ can be found in proximity of $d_{w}{ }^{*}=f_{k^{*}}$ and is therefore meaningful in search of approximation of global maximum point of function $I_{w}$ in the form of

$$
\left(k^{*}+\delta\right) \frac{1}{m \Delta t}, \delta \in\left\langle-\frac{1}{2}, \frac{1}{2}\right\rangle
$$

Whole class of methods is based on this approach, some of them described in the text below.
In Fig. 2 the discreet periodogram is given and values of global maximum $f_{w} *$ and its approximation $d_{w} *$ are shown.

\subsection{Parabola method}

In order to determine dominant frequency, parabola method is proposed in articles [11] and [12], described in this chapter.

Let $k \in\left\{0, \ldots,\left[\frac{m-1}{2}\right]\right\}$ be index for which it applies (16). Within proximity of point $f_{k^{*}}=\frac{k^{*}}{m \Delta t}$, function $I_{w}$ is approximated through three points:

$$
\left(\frac{k^{*}-1}{m \Delta t}, \mathrm{I}_{w}\left(f_{k^{*}-1}\right)\right),\left(\frac{k^{*}}{m \Delta t}, \mathrm{I}_{w}\left(f_{k^{*}}\right)\right),\left(\frac{k^{*}+1}{m \Delta t}, \mathrm{I}_{w}\left(f_{k^{*}+1}\right)\right),
$$

which equation is

$$
P(x)=a x^{2}+b x+c
$$

where it is:

$a=\frac{1}{2}\left(I_{w}\left(f_{k^{*}-1}\right)-2 I_{w}\left(f_{k^{*}}\right)+I_{w}\left(f_{k^{*}+1}\right)\right)(m \Delta t)^{2}$

$b=-\frac{1}{2}\left(\left(1+2 \mathrm{k}^{*}\right) I_{w}\left(f_{k^{*}-1}\right)-4 k^{*} I_{w}\left(f_{k^{*}}\right)-\right.$

$\left.-\left(1-2 \mathrm{k}^{*}\right) I_{w}\left(f_{k^{*}+1}\right)\right) m \Delta t$

$c=\frac{1}{2}\left(k^{*}\left(k^{*}+1\right) I_{w}\left(f_{k^{*}-1}\right)-2\left(\left(k^{*}\right)^{2}-1\right) I_{w}\left(f_{k^{*}}\right)+\right.$ Since it $\left.+\left(k^{*}-1\right) I_{w}\left(f_{k^{*}+1}\right)\right)$

is $\quad I_{w}\left(f_{k^{*}}\right) \geq I_{w}\left(f_{k}\right)$ for $\quad$ each $k \in\left\{0, \ldots,\left[\frac{m-1}{2}\right]\right\}$, therefore $I_{w}\left(f_{k^{*}}-1\right)-2 I_{w}\left(f_{k^{*}}\right)+I_{w}\left(f_{k^{*}+1}\right)=$ $=\left(I_{w}\left(f_{k^{*}-1}\right)-I_{w}\left(f_{k^{*}}\right)\right)+\left(I_{w}\left(f_{k^{*}+1}\right)-I_{w}\left(f_{k^{*}}\right)\right)<0$

namely $a<0$. Thereby quadratic function $P$ shall reach its maximum in point

$q_{w}^{*}=-\frac{b}{2 a}=\left(k^{*}+\frac{I_{w}\left(f_{k^{*}-1}\right)-I_{w}\left(f_{k^{*}+1}\right)}{2 I_{w}\left(f_{k^{*}-1}\right)-4 I_{w}\left(f_{k^{*}}\right)+2 I_{w}\left(f_{k^{*}+1}\right)}\right)$.

$\frac{1}{m \Delta t}$

and to approximate global maximum point $f_{w} *$ of function $I_{w}$, according to this approach we take point $q_{w} *$ . Aware of parabola method approximation $q_{w} *$, global maximum of function $I_{w}$ is in form (18) while

$$
\delta=\frac{I_{w}\left(f_{k^{*}-1}\right)-I_{w}\left(f_{k^{*}+1}\right)}{2 I_{w}\left(f_{k^{*}-1}\right)-4 I_{w}\left(f_{k^{*}}\right)+2 I_{w}\left(f_{k^{*}+1}\right)} \in\left\langle-\frac{1}{2}, \frac{1}{2}\right\rangle
$$

Approximation of global maximum using parabola method is shown in Fig. 3, parabola was drawn through 
three points, the maximum of discretized function and two neighbouring points, and approximation $q_{w} *$ is shown.

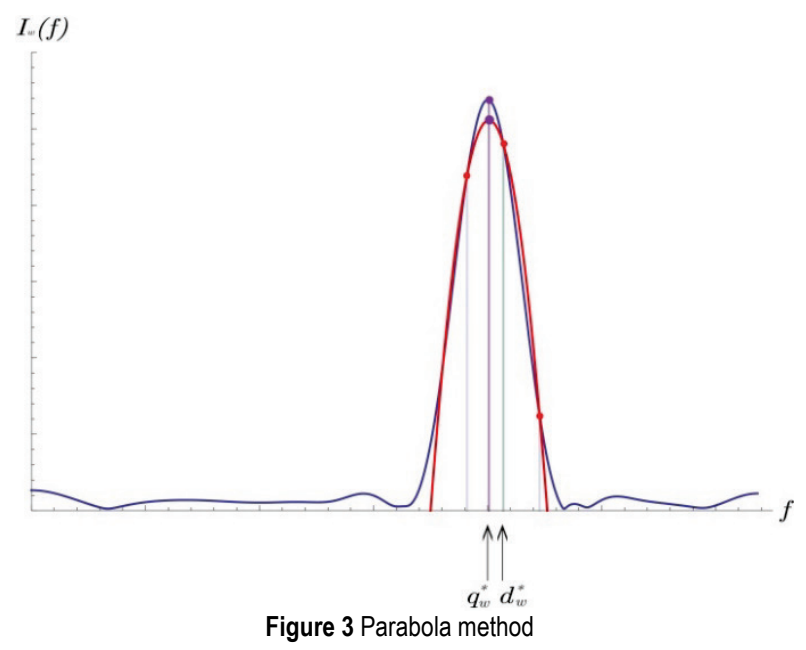

Approximations for amplitude and matching phase shift are obtained from equation system (7) and (8).

\subsection{Modified Parabola Method}

In works [13] and [14] another global maximum approximation method based on approximation of modified periodogram in case when $m$ is large enough is suggested. Below the method is formulated in analogy to previous chapter.

Let's have $k \in\left\{0, \ldots,\left[\frac{m-1}{2}\right]\right\}$ such that it applies (16). In proximity of point $f_{k^{*}}=\frac{k^{*}}{m \Delta t}$ function $I_{w}$ is also approximated by parabola through three points, while detecting two cases:

- If it applies $I_{w}\left(f_{k^{*}+1}\right)>I_{w}\left(f_{k^{*}-1}\right)$, observe parabola $P(x)=a x^{2}+b x+c$ through points

$\left(\frac{k^{*}}{m \Delta t}, \mathrm{I}_{w}\left(f_{k^{*}}\right)\right),\left(\frac{k^{*}+\frac{1}{2}}{m \Delta t}, \frac{3}{4}\left(\mathrm{I}_{w}\left(f_{k^{*}}\right)+\mathrm{I}_{w}\left(f_{k^{*}+1}\right)\right)\right)$,

$\left(\frac{k^{*}+1}{m \Delta t}, \mathrm{I}_{w}\left(f_{k^{*}+1}\right)\right)$

for which applies:

$a=-\left(I_{w}\left(f_{k^{*}+1}\right)+I_{w}\left(f_{k^{*}}\right)\right)(m \Delta t)^{2}$

$b=2\left(k^{*} I_{w}\left(f_{k^{*}}\right)+\left(1+k^{*}\right) I_{w}\left(f_{k+1}\right)\right) m \Delta t$

$c=-\left(\left(k^{*}\right)^{2}-1\right) I_{w}\left(f_{k^{*}}\right)-k^{*}\left(k^{*}+2\right) I_{w}\left(f_{k^{*}+1}\right)$

Note that $a<0$ and the quadratic function $P$ reaches maximum at point

$$
\begin{aligned}
v_{w}^{*} & =-\frac{b}{2 a}=\left(k^{*}+\frac{I_{w}\left(f_{k^{*}+1}\right)}{I_{w}\left(f_{k^{*}}\right)+I_{w}\left(f_{k^{*}+1}\right)}\right) \frac{1}{m \Delta t} \\
& =\left(k^{*}+\delta\right) \frac{1}{m \Delta t}
\end{aligned}
$$

where it is

$$
\delta=\frac{I_{w}\left(f_{k^{*}+1}\right)}{I_{w}\left(f_{k^{*}}\right)+I_{w}\left(f_{k^{*}+1}\right)} \in\left\langle-\frac{1}{2}, \frac{1}{2}\right\rangle .
$$

- If it applies $I_{w}\left(f_{k^{*}+1}\right)<I_{w}\left(f_{k^{*}-1}\right)$, observe parabola $P(x)=a x^{2}+b x+c$ matching points

$$
\begin{aligned}
& \left(\frac{k^{*}-1}{m \Delta t}, \mathrm{I}_{w}\left(f_{k^{*}}\right)\right),\left(\frac{k^{*}-\frac{1}{2}}{m \Delta t}, \frac{3}{4}\left(\mathrm{I}_{w}\left(f_{k^{*}-1}\right)+\mathrm{I}_{w}\left(f_{k^{*}}\right)\right)\right), \\
& \left(\frac{k^{*}}{m \Delta t} \mathrm{I}_{w}\left(f_{k^{*}}\right)\right)
\end{aligned}
$$

where it applies

$$
\begin{aligned}
a & =-\left(I_{w}\left(f_{k^{*}-1}\right)+I_{w}\left(f_{k^{*}}\right)\right)(m \Delta t)^{2} \\
b & =2\left(k^{*} I_{w}\left(f_{k^{*}}\right)+\left(k^{*}-1\right) I_{w}\left(f_{k^{*}-1}\right)\right) m \Delta t \\
c & =-\left(\left(k^{*}\right)^{2}-1\right) I_{w}\left(f_{k^{*}}\right)-k^{*}\left(k^{*}-2\right) I_{w}\left(f_{k^{*}-1}\right) \\
v_{w}^{*} & =-\frac{b}{2 a}=\left(k^{*}-\frac{I_{w}\left(f_{k^{*}-1}\right)}{I_{w}\left(f_{k^{*}}\right)+I_{w}\left(f_{k^{*}-1}\right)}\right) \frac{1}{m \Delta t} \\
& =\left(k^{*}+\delta\right) \frac{1}{m \Delta t}
\end{aligned}
$$

where it is:

$\delta=-\frac{I_{w}\left(f_{k^{*}-1}\right)}{I_{w}\left(f_{k^{*}}\right)+I_{w}\left(f_{k^{*}-1}\right)} \in\left\langle-\frac{1}{2}, \frac{1}{2}\right\rangle$

Finally for approximation of global maximum point $f_{w}{ }^{*}$ of function $I_{w}$, based on this approach we take point $v_{w}^{*}=(k *+\delta) \frac{1}{m \Delta t}$,

where it is

$\delta=\left\{\begin{array}{l}\frac{I_{w}\left(f_{k^{*}+1}\right)}{I_{w}\left(f_{k^{*}}\right)+I_{w}\left(f_{k^{*}+1}\right)}, I_{w}\left(f_{k^{*}+1}\right)>I_{w}\left(f_{k^{*}-1}\right) \\ -\frac{I_{w}\left(f_{k^{*}-1}\right)}{I_{w}\left(f_{k^{*}}\right)+I_{w}\left(f_{k^{*}-1}\right)}, I_{w}\left(f_{k^{*}+1}\right) \leq I_{w}\left(f_{k^{*}-1}\right)\end{array}\right.$

$\delta=\left\{\begin{array}{l}\frac{1}{\frac{I_{w}\left(f_{k^{*}}\right)}{I_{w}\left(f_{k^{*}+1}\right)}+1}, \frac{I_{w}\left(f_{k^{*}+1}\right)}{I_{w}\left(f_{k^{*}-1}\right)}>1 \\ -\frac{1}{\frac{I_{w}\left(f_{k^{*}}\right)}{I_{w}\left(f_{k^{*}-1}\right)}+1}, \frac{I_{w}\left(f_{k^{*}+1}\right)}{I_{w}\left(f_{k^{*}-1}\right)} \leq 1\end{array}\right.$ 
Approximations for amplitude and matching phase shift are obtained from equation system (7) and (8).

\subsection{Quinn's Period Maximization Method}

Two more effective methods for determination of dominant frequencies are suggested in articles [14] and [15]. Without step by step formulation and additional analysis we list these two methods.

$$
\delta=\left\{\begin{array}{c}
\frac{I_{w}\left(f_{k^{*}+1}\right)}{I_{w}\left(f_{k^{*}}\right)+I_{w}\left(f_{k^{*}+1}\right)} \\
\frac{\operatorname{sign}\left(\operatorname{Re}\left(Y_{w}\left(f_{k^{*}-1}\right) \overline{Y_{w}\left(f_{k^{*}}\right)}\right)\right) I_{w}\left(f_{k^{*}-1}\right)}{I_{w}\left(f_{k^{*}}\right)-\operatorname{sign}\left(\operatorname{Re}\left(Y_{w}\left(f_{k^{*}-1}\right) \overline{Y_{w}\left(f_{k^{*}}\right)}\right)\right) I_{w}\left(f_{k^{*}-1}\right)}
\end{array}\right.
$$$$
\operatorname{sign}\left(\operatorname{Re}\left(Y_{w}\left(f_{k^{*}+1}\right) \overline{Y_{w}\left(f_{k^{*}}\right)}\right)\right)=-1 \&
$$$$
\operatorname{sign}\left(\operatorname{Re}\left(Y_{w}\left(f_{k^{*}-1}\right) \overline{Y_{w}\left(f_{k^{*}}\right)}\right)\right)=1
$$

otherwise $I_{w}(f)=\left|Y_{w}(f)\right|$.

$$
\beta_{w}^{*}=\left(k^{*}+\delta\right) \frac{1}{m \Delta t}
$$

where it applies
Method Quinn 1994 [15]: dominant frequency approximation is

$\beta_{w}^{*}=\left(k^{*}+\delta\right) \frac{1}{m \Delta t}$

where it applies

$$
\delta=\left\{\begin{array}{cc}
\frac{I_{w}\left(f_{k^{*}+1}\right)}{\frac{I_{w}}{}\left(f_{k^{*}+1}\right)} & \frac{I_{w}\left(f_{k^{*}+1}\right)-I_{w}\left(f_{k^{*}}\right)}{I_{w}\left(f_{k^{*}+1}\right)-I_{w}\left(f_{k^{*}}\right)} \\
& \frac{I_{w}\left(f_{k^{*}-1}\right)}{I_{w}\left(f_{k^{*}}\right)-I_{w}\left(f_{k^{*}-1}\right)}>0 \& \\
\frac{I_{w}\left(f_{k^{*}-1}\right)}{I_{w}\left(f_{k^{*}}\right)-I_{w}\left(f_{k^{*}-1}\right)} & \text { otherwise }
\end{array}\right.
$$

Approximations for amplitude and matching phase shift are obtained from equation system (7) and (8).

\section{GLOBAL METHOD FOR DOMINANT FREQUENCY ESTIMATION}

Function $A_{w}$ maximization problem is equal to the following function minimization problem

$$
\min _{f \in\left[0, \frac{1}{2 \Delta t}\right]}-A_{w}(f)
$$

It is shown that the function $-A_{w}$ is Lipschitz function at $\mathbb{R}$, namely there is constant $L>0$ such that for $\alpha, \beta \in \mathbb{R}$ applies

$$
\left|A_{w}(\beta)-A_{w}(\alpha)\right| \leq L|\beta-\alpha|
$$

Proposition. Function $-A_{w}:\left\langle 0, \frac{1}{2 \Delta t}\right\rangle \rightarrow \mathbb{R}$ is Lipschitz at $\mathbb{R}$.

Proof. Since $-A_{w}$ is derivable at $\mathbb{R}$ it is sufficient to show
Let it be $Y_{w}(f)=\frac{1}{m} \sum_{n=0}^{m-1} w_{n} y_{n} e^{-2 n \pi i f \Delta t}$, notice that it is

Method Quinn 1997 [14]: dominant frequency approximation is

$$
\begin{aligned}
& \left|A^{\prime}{ }_{w}(f)\right|=\frac{1}{m} \mid-4 \pi \Delta t \sum_{n=0}^{m-1} w_{n} y_{n} \cos (2 \pi f n \Delta t) \\
& \sum_{n=0}^{m-1} n w_{n} y_{n} \sin (2 \pi f n \Delta t)+ \\
& +4 \pi \Delta t \sum_{n=0}^{m-1} w_{n} y_{n} \sin (2 \pi f n \Delta t) \sum_{n=0}^{m-1} n w_{n} y_{n} \cos (2 \pi f n \Delta t) \mid \\
& \leq \frac{8 \pi \Delta t}{m} \sum_{n=0}^{m-1} n w_{n}\left|y_{n}\right| \sum_{n=0}^{m-1} w_{n}\left|y_{n}\right|=: L
\end{aligned}
$$

it follows $\alpha, \beta \in R$ exists $\varsigma \in\langle\alpha, \beta\rangle$ such that

$$
\left|A_{w}(\beta)-A_{w}(\alpha)\right|=\left|A_{w}(\varsigma)\right||\beta-\alpha| \leq L|\beta-\alpha|
$$

namely the function $-A_{w}$ is Lipschitz function.

As function $-A_{w}$ is Lipschitz class function at $\mathbb{R}$, it follows that it's Lipschitz at $\left[0, \frac{1}{2 \Delta t}\right]$ and accordingly problem (35) can be solved applying DIRECT method for global minimization of Lipchitz function $[7,8,9,16]$.

Instead of minimization of function $-A_{w}$ at whole interval, and applying DIRECT method function at function $-A_{w}$, it is meaningful to minimize function only in proximity of point $f_{k^{*}}=\frac{k^{*}}{m \Delta t}$, where it is $k^{*} \in\left\{0, \ldots,\left[\frac{m-1}{2}\right]\right\}$ such that it applies (16). Motivated by (18) function $-A_{w}$ is minimized on interval $\left[f_{k^{*}}-\frac{1}{2 m \Delta t}, f_{k^{*}}+\frac{1}{2 m \Delta t}\right]$, namely instead of (35) we solve minimization problem on smaller domain.

$$
f \in\left[f_{k^{*}-\frac{1}{2 m \Delta t}} f_{k^{*}+\frac{1}{2 m \Delta t}}\right]^{-A_{w}(f)}
$$




\section{DETERMINATION OF REMAINING DOMINANT FREQUENCIES - MULTIPEAK DETECTION}

To determine remaining subsequent dominant frequencies by filtering global maximum in this article is used inverse Fourier transform, can also be used digital filter as shown in [17].

Several methods are described in above sections which can be used to estimate dominant frequency or global maximum, and afterwards using equation system (7) and (8) to obtain amplitude and phase shift.

There are several methods for multiple peak detection, depending on the signal origin and physical meaning eg. [18-20]. Also online can be found many solutions for multiple peak detection; some examples are given in $[21$, 22].

Here the authors propose the following algorithm to estimate remaining dominant frequencies and their matching amplitudes:

\subsection{Dominant frequencies determination algorithm}

1. Assign

i) $L>0, \quad m \in \mathbb{N}, \quad \Delta t=\frac{L}{m} \quad$, data $\left(t_{m}, y_{n}\right)$, $n=0, \ldots, m-1$

ii) window function $w:[0, L] \rightarrow[0, \infty\rangle$

iii) number of obvious dominant frequencies

2. Determine dominant frequency $f_{w} *(1)$ using one of the metods described in sections 3 and 4 and matching amplitude $a_{w} *(1)$ and phase shift $\varphi_{w} *(1)$ based on system of Eq. (7) and (8).

3 . for $i=2$ to $\kappa$

Redefine data $\left(t_{n}, x_{n}\right), n=0, \ldots ., m-1$ where it applies $y_{n}=y_{n}-a_{w} *(i-1) \cos \left(2 \pi f_{w} *(i-1) n \Delta t+\varphi_{w} *(i-1)\right)$

end

4. Write

i) dominant frequencies $f_{w} *(j), j=1, \ldots, \kappa$

ii) amplitudes: $a_{w} *(j), j=1, \ldots, \kappa$

iii) phase shifts: $\varphi_{w} *(j), j=1, \ldots, \kappa$

\section{NUMERICAL EXPERIMENT}

In following sections are given results of two numerical experiment examples. Experiment was done using numerical procedures.

\subsection{Example 1}

Let us state $L>0, m \in \mathbb{N}, \Delta t=\frac{L}{m}$, data $\left(t_{n}, y_{n}\right)$, $n=0, \ldots, m-1$ is generated such that it applies

$t_{n}=n \Delta t, y_{n}=11 \cos (2 \pi \cdot 1 n \Delta t+1.1)+\varepsilon_{n}$

where it is $\varepsilon_{n} \sim N\left(0, \sigma^{2}\right)$.

Let it be $m=50$ and $\sigma \in\{0.1,0.5,0.9,1.7,2.1,2.5\}$ for some random set of input data using the above described methods: Discreet Fourier transformation discretization of periodogram (DFT), Parabola method 1 (P1), Modified parabola method (P2), Method Quinn 1997 (Q97), Method Quinn 1994 (Q94), Global method (GM) we estimate frequency $f_{j}$ as well as matching amplitude $a_{j}$ and phase shift $\varphi_{j}$.

For all methods reconstructed signal will be compared to the original signal. Results of frequency, amplitude and phase are given in Tabs. 1, 2 and 3 for rectangular window function and Tabs. 4, 5, 6 for Hanning window function.

Table 1 Results of frequencies for rectangular window function

\begin{tabular}{|c|c|c|c|c|c|c|}
\hline & discreet & parabola & parabola 2 & Quinn 97 & Qiunn 94 & Lipschitz \\
\hline Sigma $=0.1$ & 0.00203134 & 0.00127251 & 0.0000288497 & 0.0000257985 & 0.0000288497 & 0.0000005725 \\
\hline Sigma $=0.5$ & 0.00203134 & 0.00126982 & 0.0000359473 & 0.0000318505 & 0.0000359473 & 0.0000024904 \\
\hline Sigma $=0.9$ & 0.240226 & 0.00127108 & 0.0000501181 & 0.0000436677 & 0.0000501181 & 0.00000699837 \\
\hline Sigma $=1.3$ & 0.764254 & 0.00127682 & 0.0000713641 & 0.000061164 & 0.0000713641 & 0.0000141129 \\
\hline Sigma $=1.7$ & 1.05009 & 0.00128762 & 0.000180505 & 0.0000842825 & 0.0000996327 & 0.0000238564 \\
\hline Sigma $=2.1$ & 1.193 & 0.00130371 & 0.000551392 & 0.000133457 & 0.000162137 & 0.0000362577 \\
\hline Sigma $=2.5$ & 1.24064 & 0.00132435 & 0.00100511 & 0.000194253 & 0.00026484 & 0.000051352 \\
\hline
\end{tabular}

Table 2 Results of amlitudes for rectangular window function

\begin{tabular}{|c|c|c|c|c|c|c|}
\hline & discreet & parabola & parabola 2 & Quinn 97 & Qiunn 94 & Lipschitz \\
\hline Sigma $=0.1$ & 0.398648 & 0.24326 & 0.142554 & 0.141606 & 0.142554 & 0.130874 \\
\hline Sigma $=0.5$ & 0.400135 & 0.246319 & 0.147337 & 0.146143 & 0.147337 & 0.13522 \\
\hline Sigma $=0.9$ & 0.403327 & 0.26292 & 0.164232 & 0.162515 & 0.164232 & 0.151555 \\
\hline Sigma $=1.3$ & 0.400706 & 0.293156 & 0.193195 & 0.190721 & 0.193195 & 0.179891 \\
\hline Sigma $=1.7$ & 0.390318 & 0.337149 & 0.257849 & 0.230771 & 0.234196 & 0.220247 \\
\hline Sigma $=2.1$ & 0.382874 & 0.395006 & 0.386669 & 0.282555 & 0.287539 & 0.272642 \\
\hline Sigma $=2.5$ & 0.388103 & 0.466728 & 0.567863 & 0.349037 & 0.362886 & 0.337097 \\
\hline
\end{tabular}

Table 3 Results of phase for rectangular window function

\begin{tabular}{|c|c|c|c|c|c|c|}
\hline & discreet & parabola & parabola 2 & Quinn 97 & Qiunn 94 & Lipshitz \\
\hline Sigma $=0.1$ & 0.312287 & 0.15167 & 0.00480191 & 0.00441043 & 0.00480191 & 0.000148262 \\
\hline Sigma $=0.5$ & 0.312864 & 0.151927 & 0.00560693 & 0.00510285 & 0.00560693 & 0.0004413 \\
\hline Sigma $=0.9$ & 0.331657 & 0.152792 & 0.00727609 & 0.0065192 & 0.00727609 & 0.00113917 \\
\hline Sigma $=1.3$ & 0.370176 & 0.154322 & 0.00980576 & 0.0086512 & 0.00980576 & 0.00224395 \\
\hline Sigma $=1.7$ & 0.393201 & 0.156578 & 0.0210787 & 0.0114934 & 0.0131873 & 0.00375796 \\
\hline Sigma $=2.1$ & 0.403089 & 0.159581 & 0.059402 & 0.01707 & 0.0200951 & 0.00568383 \\
\hline Sigma $=2.5$ & 0.408133 & 0.163253 & 0.10395 & 0.0239329 & 0.0313072 & 0.00802452 \\
\hline
\end{tabular}


Table 4 Results of frequencies for Hanning window function

\begin{tabular}{|c|c|c|c|c|c|c|}
\hline & discreet & parabola & parabola 2 & Quinn 97 & Qiunn 94 & Lipshitz \\
\hline Sigma $=0.5$ & 0.00203134 & 0.00010653 & 0.00603202 & 4.22485 & 4.22217 & 0.00000024904 \\
\hline Sigma $=0.9$ & 0.00203134 & 0.000119886 & 0.0060449 & 4.22496 & 4.22214 & 0.00000699837 \\
\hline Sigma $=1.3$ & 0.00203134 & 0.000142451 & 0.00605948 & 4.05723 & 4.09624 & 0.0000141129 \\
\hline Sigma $=1.7$ & 0.00203134 & 0.000174348 & 0.00607577 & 3.97363 & 3.97057 & 0.0000238564 \\
\hline Sigma $=2.1$ & 0.00203134 & 0.000215749 & 0.00630649 & 3.72112 & 3.71798 & 0.0000362577 \\
\hline Sigma $=2.5$ & 0.0844243 & 0.000266876 & 0.0063421 & 3.468 & 3.46469 & 0.000051352 \\
\hline
\end{tabular}

Table 5 Results of amlitudes for Hanning window function

\begin{tabular}{|c|c|c|c|c|c|c|}
\hline & discreet & parabola & parabola 2 & Quinn 97 & Qiunn 94 & Lipshitz \\
\hline Sigma $=0.1$ & 0.398648 & 0.118049 & 2.06786 & 16.4745 & 16.503 \\
\hline Sigma $=0.5$ & 0.400135 & 0.123353 & 2.05865 & 16.4914 & 16.529 \\
\hline Sigma $=0.9$ & 0.414848 & 0.14168 & 2.05674 & 16.5193 & 16.5763 & 0.13522 \\
\hline Sigma $=1.3$ & 0.442788 & 0.173054 & 2.06224 & 16.2386 & 16.3771 & 0.151555 \\
\hline Sigma $=1.7$ & 0.483952 & 0.217533 & 2.07527 & 16.1683 & 16.2876 & 0.179891 \\
\hline Sigma $=2.1$ & 0.538329 & 0.275203 & 2.16779 & 15.8613 & 16.0274 & 0.220247 \\
\hline Sigma $=2.5$ & 0.605403 & 0.346182 & 2.2008 & 15.5771 & 15.806 & 0.337097 \\
\hline
\end{tabular}

Table 6 Results of phase for Hanning window function

\begin{tabular}{|c|c|c|c|c|c|c|}
\hline & discreet & parabola & parabola 2 & Quinn 97 & Qiunn 94 & Lipshitz \\
\hline Sigma $=0.1$ & 0.312287 & 0.00746186 & 0.616335 & 0.179689 & 0.185053 \\
\hline Sigma $=0.5$ & 0.312864 & 0.00800005 & 0.616567 & 0.180251 & 0.185757 \\
\hline Sigma $=0.9$ & 0.313583 & 0.00943011 & 0.617129 & 0.18089 & 0.0004413 \\
\hline Sigma $=1.3$ & 0.314443 & 0.0117661 & 0.618018 & 0.267009 & 0.18668 & 0.00113917 \\
\hline Sigma $=1.7$ & 0.315442 & 0.0150281 & 0.619226 & 0.312917 & 0.319446 & 0.00375796 \\
\hline Sigma $=2.1$ & 0.316581 & 0.0192429 & 0.643179 & 0.440763 & 0.448251 & 0.00568383 \\
\hline Sigma $=2.5$ & 0.370351 & 0.024445 & 0.647252 & 0.552666 & 0.56261 & 0.00802452 \\
\hline
\end{tabular}

Discretized original signal with random error

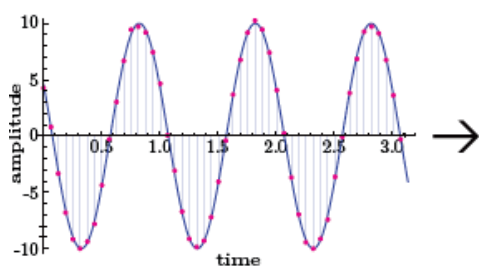

$A_{-}(f)$

Periodogram

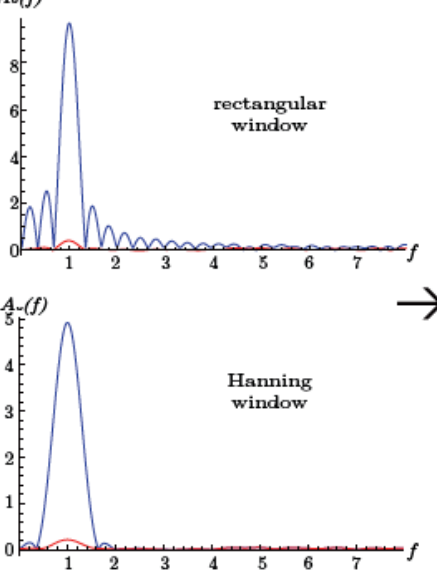

Figure 4 Signal reconstruction
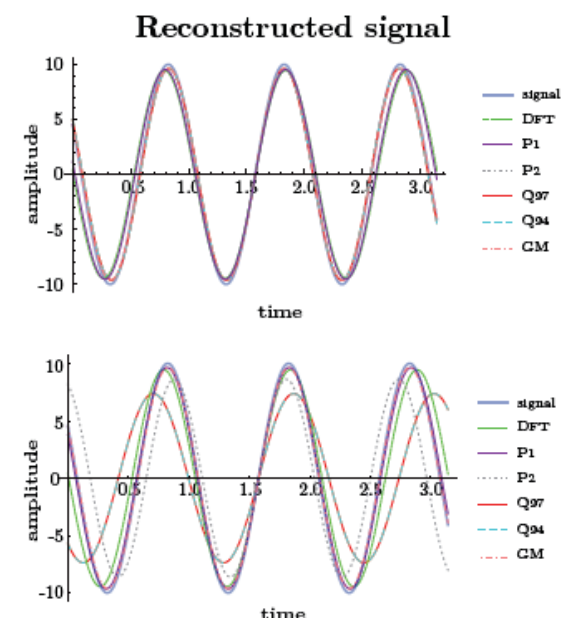

\subsection{Example 2}

Let it be $L>0 L>0, m \in \mathbb{N}, \Delta t=\frac{L}{m}$, data $\left(t_{n}, y_{n}\right)$, $n=0, \ldots, m-1$ is generated so that $t_{n}=n \Delta t$, $y_{n}=11 \cos \left(2 \pi \cdot 4 n \Delta t+\frac{\pi}{2}\right)+8 \cos (2 \pi \cdot 2 n \Delta t+\pi)+\varepsilon_{n}$

where $\varepsilon_{n} \sim N\left(0, \sigma^{2}\right)$. Let it be $m=50$. For each $\sigma \in\{0.1,0.5,0.9,1.7,2.1,2.5\}$ the experiment is repeated 100 times. Applying above mentioned methods: Discreet Fourier transformation - discretization of periodogram (DFT), Parabola method 1 (P1), Modified parabola method (P2), Method Quinn 1997 (Q97), Method Quinn 1994 (Q94), Global method (GM) we estimate frequency $f_{j}$ as well as matching amplitude $a_{j}$ and phase shift $\varphi_{j}$. For each method average error is calculated:
- for frequency $E_{f}=\frac{1}{100} \sum_{j=i}^{100} \frac{\left|4-\hat{f}_{j}\right|}{\hat{f}_{j}}$

- for amplitude $E_{a}=\frac{1}{100} \sum_{j=i}^{100} \frac{\left|4-\hat{a}_{j}\right|}{\hat{a}_{j}}$

- for phase $E_{\varphi}=\frac{1}{100} \sum_{j=i}^{100} \frac{\left|4-\hat{\varphi}_{j}\right|}{\hat{\varphi}_{j}}$

Experiment was conducted with Hanning and rectangular window function, in Fig. 5 are shown the results. After isolating first dominant frequency it is obvious that frequency spectrum is smoothed and additionally emphasized using Hanning window function.

Results obtained while conducting numerical experiment are shown in Figs. 6 and 7. Most precise is DIRECT global method, with slightly less accuracy at calculating second dominant frequency. 

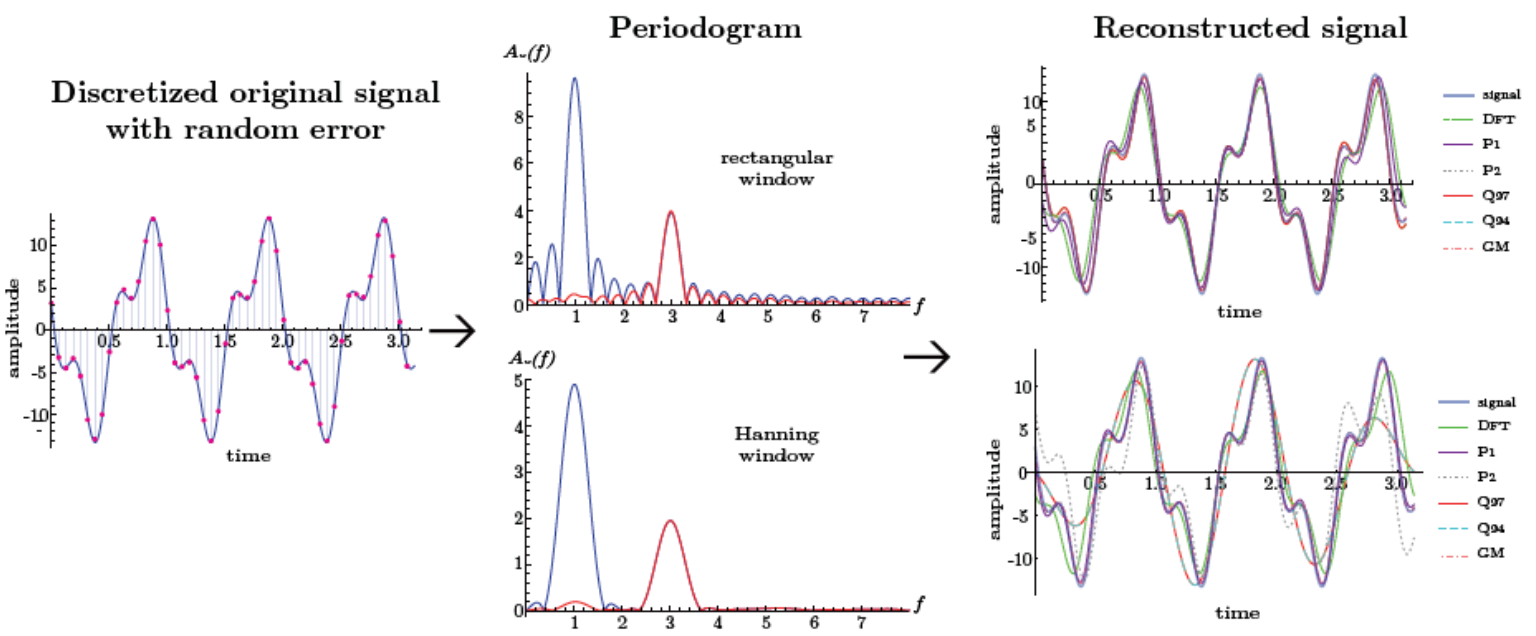

Figure 5 Signal reconstruction after obtaining two dominant frequencies
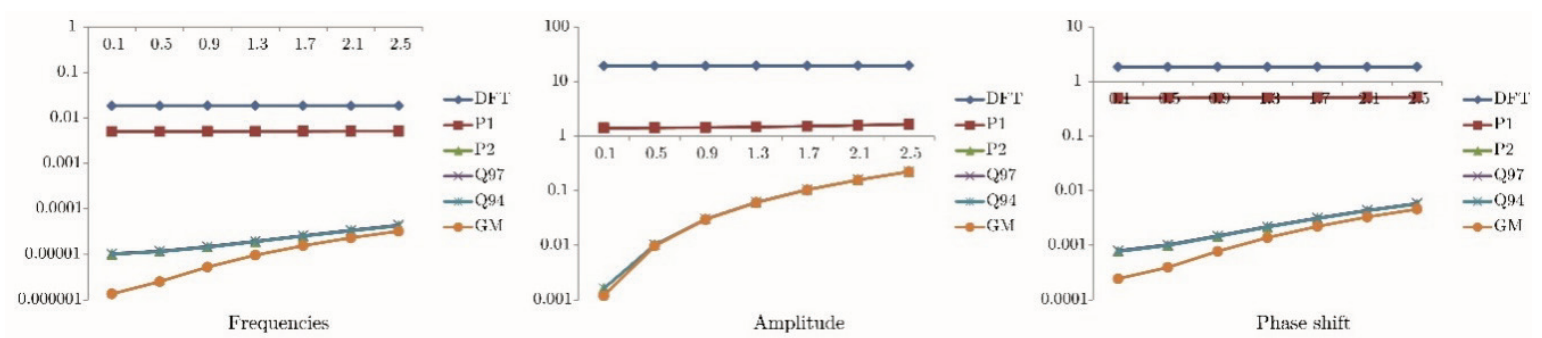

Relative error of first dominant frequency results
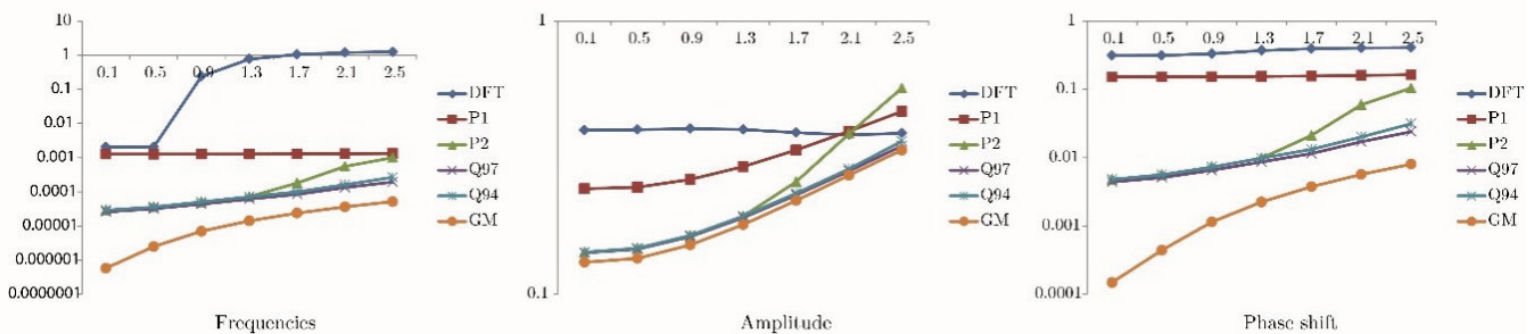

Relative error of second dominant frequency results

Figure 6 Comparison of results obtained using rectangular window function

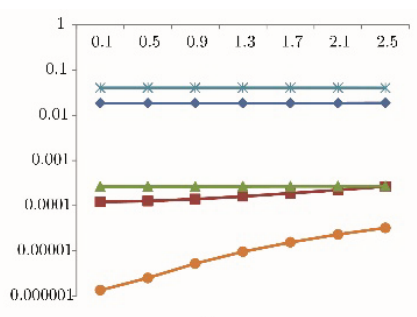

Frequencios

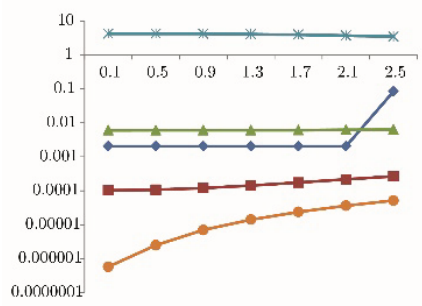

Frequencies
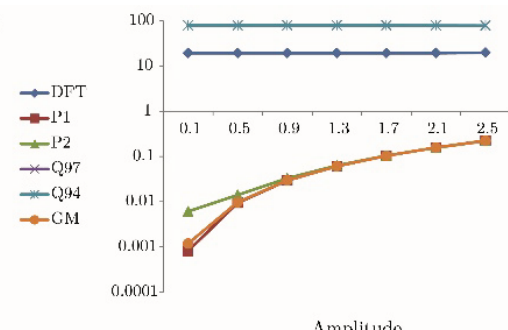

Amplitude

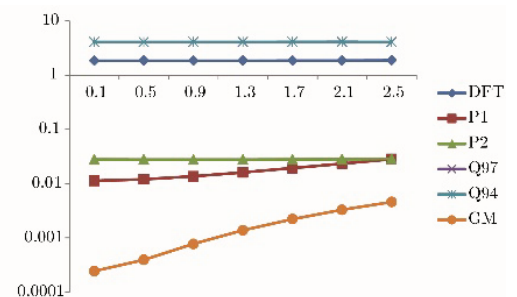

Phase shift

Relative error of first dominant frequency results

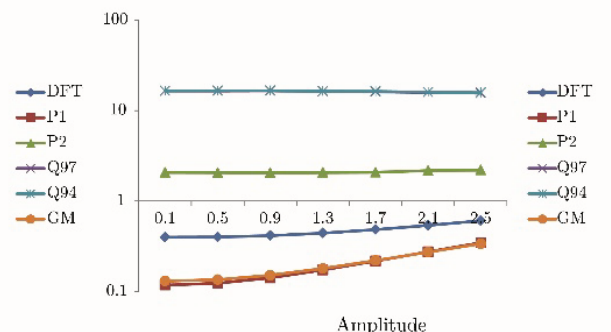

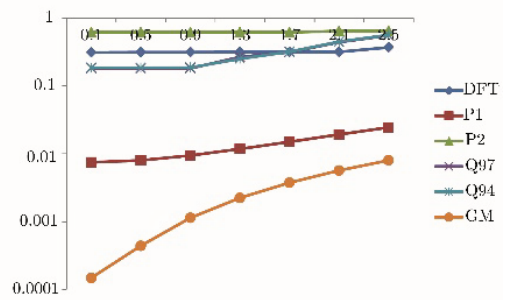

Phase shift

Relative error of second dominant frequency results

Figure 7 Comparison of results obtained using Hanning window function

Comparison of results shows that more accurate results are obtained using Hanning window function at larger data dissipation, but for more accurate assessment algorithm should be tested on real measurements data or more realistic data should be obtained such as calculated using finite element method. 
Testing input functions has shown that in case when dominant frequencies are sufficiently distant, after isolating dominant frequency from periodogram, algorithm can be effectively used to obtain subsequent dominant frequency. If dominant frequencies are in vicinity then inverse transformation of reduced periodogram gives slightly different result which has larger deviation from real value of maximum.

\section{CONCLUSION}

Comparing results of testing methods for vibration dominant frequencies determination, conclusion can be drawn that global method is most accurate in determination of frequencies, amplitude and phase shift in the experiment where rectangular window was used. While using Hanning window DIRECT global method and parabola method give similar results for amplitude but for frequency and phase shift DIRECT global method gives the most accurate results. So overall conclusion that this efficiency analysis shows can be that the global method is more accurate than some previously commonly used methods for frequency estimation.

Method efficiency varies with using different window functions. Further testing shows that algorithm efficiency is questionable at some ratios of amplitude against frequency (when frequency peeks are in vicinity), in some cases Hanning window function causes smoothing of spectra and making frequency isolation more difficult. This problem can be solved by changing sampling ratio and applying rectangular window function.

Proposed algorithm for multi peak detection could be used as part of intelligent fault detection system for rotary machines. In further research algorithm could be used to build more complex computer software and after numerical simulation, results should be tested on experimental rig and real measurement data.

\section{REFERENCES}

[1] Scheffer, C. \& Girdhar, P. (2004). Practical Machinery Vibration Analysis and Predictive Maintenance, Newnes, An imprint of Elsevier, Burlington. https://doi.org/10.1016/B978-075066275-8/50002-3

[2] San-Millan, A. \& Feliu, V. (2015). A Fast Online Estimator of the Two Main Vibration Modes of Flexible Structures from Biased and Noisy Measurements. IEEE/ASME Transactionson Mechatronics, 1, 93-104. https://doi.org/10.1109/TMECH.2014.2304302

[3] Sun, Y., Zhuang, C., \& Xiong, Z. (2015). A Scale FactorBased Interpolated DFT for Chatter Frequency Estimation. IEEE Transactions on Instrumentation and Measurement, 64(10), 2666-2678. https://doi.org/10.1109/TIM.2015.2421711

[4] So, H. C., Chan, Y. T., Ma Q., \& Ching, P. C. (1999). Comparison of various periodograms for sinusoid detection and frequency estimation. IEEE Transactions on Aerospace and Electronic Systems, 35, 945-952. https://doi.org/10.1109/7.784064

[5] Rice, J. A. \& Rosenblatt, M. (1988). On frequency estimation. Biometrika, 75(3), 477-848. https://doi.org/10.1093/biomet/75.3.477

[6] Smith, S. W. (1997). The Scientist and Engineer's Guide to Digital Signal Processing. California Technical Pub, San Diego.
[7] Finkel, D. E. \& Kelley, C. T. (2004). Convergence analysis of the DIRECT algorithm.

[8] Finkel, D. E. \& Kelley, C. T. (2006). Additive scaling and the DIRECT algorithm. Journal of Global Optimisation, 36597-608.

[9] Jones, D. R., Perttunen, C. D., \& Stuckman, B. E. (1993). Lipschitzian optimization without the Lipschitz constant. Journal of Optimization Theory and Applications, 79, 157-181. https://doi.org/10.1007/BF00941892

[10] Plato, R. (2003).Concise numerical mathematics. American Mathematical Society, Providence.

[11] Jacobsen, E. \& Kootsookos, P. (2007). Fast, Accurate Frequency Estimators. IEEE Signal Processing Magazine, 24, 123-125. https://doi.org/10.1109/MSP.2007.361611

[12] Voglewede, P. (2004). Parabola approximation for peak determination. Global DSP Magazine, 3, 13-17.

[13] Rife, D. C. \& Vincent, G. A. (1970). Use of the discrete Fourier transform in the measurement of frequencies and levels of tones. The Bell System Technical Journal, 49(2), 197-228. https://doi.org/10.1002/j.1538-7305.1970.tb01766.x

[14] Quinn, B. G. (1997). Estimation of Frequency, Amplitude and Phase from the DFT of a Time Series. IEEE Transactions on Signal Processing, 45(5), 814-817. https://doi.org/10.1109/78.558515

[15] Quinn, B. G. (1994). Estimating frequency by interpolation using Fourier coefficients. IEEE Transactions on Signal Processing, 42(5), 1265-1268. https://doi.org/10.1109/78.295186

[16] Wood, G. R. \& Zhang, B.P. (1996). Estimation of the Lipschitz constant of a function. Journal of Global Optimization, 8(1), 91-103. https://doi.org/10.1007/BF00229304

[17] Wang, Y. F. \& Kootsookos, P. J. (1997). Frequency Estimation in the Fault Detection of Rolling Element Bearing. Fifth International Congress on Sound and Vibration, Adelaide, 1073-1080.

[18] Yu, W., He, Z., Liu, Y., \& Zhao, H. (2008). Improving Mass Spectrometry Peak Detection Using Multiple Peak Alignment Results. Journal of Proteome Research, 7(1), 123-129. https://doi.org/10.1021/pro70370n

[19] Chen, Y., Yang, K., \& Liu, H. L. (2016). Self-Adaptive Multi-Peak Detection Algorithm for FBG Sensing Signal, IEEE Sensors Journal, 16(8), 2658-2665. https://doi.org/10.1109/JSEN.2016.2516038

[20] Scholkmann, F., Boss, J., \& Wolf, M. (2012). An Efficient Algorithm for Automatic Peak Detection in Noisy Periodic and Quasi-Periodic Signals. Algorithms, 5, 588-603. https://doi.org/10.3390/a5040588

[21] Wave metrics. Multi peak fitting. Retrieved from https://www.wavemetrics.com/products/igorpro/dataanalysi s/peakanalysis/multipeakfitting

[22] Findpeaks. Find local maxima. Retrieved from https://www.mathworks.com/help/signal/ref/findpeaks.html

\section{Contact information}

Katarina PISAČIĆ, dipl. ing. mech., Senior Lecturer (Corresponding author)

University North, University center Varaždin

104. brigade 3, 42000 Varaždin, Croatia

E-mail: katarina.pisacic@unin.hr

Zlatko BORAK, PhD, Assistant Professor

University North, University center Varaždin,

104. brigade 3, 42000 Varaždin, Croatia

E-mail: zlatko.botak@unin.hr

Marko HORVAT, dipl. ing. mech., Lecturer University North, University center Varaždin, 104. brigade 3, 42000 Varaždin, Croatia

E-mail: marko.horvat@unin.hr 